24 
PETER BROCKMEIER

\section{Lust und Herrschaft}

Studien über gesellschaftliche Aspekte

der Novellistik: Boccaccio, Sacchetti,

Margarete von Navarra, Cervantes

J. B. METZLERSCHE VERLAGSBUCHHANDLUNG STUTTGART 
Margitta als Dank zUgeeignet

(c) Springer-Verlag GmbH Deutschland 1972

Ursprünglich erschienen bei J. B. Metzlersche Verlagsbuchhandlung und Carl Ernst Poeschel Verlag GmbH in Stuttgart 1972.

ISBN 978-3-476-00248-8

ISBN 978-3-476-03004-7 (eBook)

DOI 10.1007/978-3-476-03004-7 


\section{INHALT}

Einleitung

IX

I. Die Liberalität der Großbürger

Giovanni Boccaccios Decameron I

1. Entrückung ins Wohlgefallen

2. Fortuna, Cortesia, Liberalità 7

3. Die Grenzen der allmächtigen Liebe 21

4. Friedliche Konkurrenz 35

Exkurs

Die Wirklichkeit der Stadtgesellschaft in Franco Sacchettis

Trecentonovelle 40

1. Die Herrschaft der Signori 40

2. Eitelkeit und Geiz 45

3. Der tückische Zufall 50

II. Höfischer Ehrenhandel

Das Heptaméron der Margarete von Navarra 54

1. Ein höfischer Liebeshandel: die III. Novelle des Heptuméron 54

2. 'Es gehört sich nicht' 59

3. Die Ehre der Kurtisane 65

4. Die 'Estimer 69

5. Mokante Konversation 76

III. Reich und Arm

Die Novelas ejemplares von Cervantes $\quad 83$

1. Der Niedergang Spaniens $\quad 83$

2. Die tragische Einsamkeit $\quad 85$

3. Das irdische und das himmlische Gesetz 92

4. Erfahrung im Labyrinth $\quad 98$

5. Poesie und Prosa 104

6. Pikareskes Utopia 111 
$\begin{array}{lr}\text { Anmerkungen } & 118\end{array}$

$\begin{array}{lr}\text { Anmerkungen zur Einleitung } & 118\end{array}$

$\begin{array}{lr}\text { Anmerkungen zu I. } & 118\end{array}$

Anmerkungen zum Exkurs $\quad 126$

Anmerkungen zu II. $\quad 129$

$\begin{array}{ll}\text { Anmerkungen zu III. } & 137\end{array}$

$\begin{array}{lr}\text { Bibliographie } & 148\end{array}$ 


\section{Abkürzungen}

Dec. = G. Boccaccio, Decameron. - Zur Zitierweise: Römische Ziffern bezeichnen den Erzähltag, arabische die Novelle dieses Tages; arabische Ziffern, die durch ein Komma von diesen beiden Angaben bzw. von den Angaben "Conclusione dell' autore", "Introduzione*, *I (etc.) conclusione* getrennt sind, beziehen sich auf die in V. Brancas Ausgabe numerierten Perioden der jeweiligen Novelle oder eines anderen Abschnitts der Sammlung.

Hept. = Marguerite de Navarre, Heptaméron. Die römischen Ziffern bezeichnen die Novellen.

N.E. $\quad=$ Cervantes, Novelas ejemplares.

O.C. = Cervantes, Obras completas.

R.M. = Cervantes, Don Quijote, hg. v. F. Rodríguez Marín. Die Zitate aus dem $»$ Don Quijote « bezeichnen zuerst $(1,33)$ Teil und Kapitel des Romans, dann (R.M. III, S. 171) Band und Seitenzahl dieser Ausgabe.

Trecnov. = F. Sachetti, Il Trecentonovelle. Die römischen Ziffern bezeichnen die Novellen.

Die Ubersetzungen aus dem Italienischen, Französischen und Spanischen, deren Ubersetzer nicht jeweils namentlich genannt werden, stammen vom Verfasser der vorliegenden Arbeit. 


\section{Einleitung}

Die Nachricht ist das Medium des Handels, und gute Neuigkeit bedeutet dem Bürger seit je Profit und Vergnügen. Üppige Sammlungen von hundert oder gar dreihundert Novellen begleiten den Aufstieg des städtischen Bürgertums seit dem ausgehenden Mittelalter; der Gattungsname novella meinte die Nachricht, die Neuigkeit. Die kurze Erzählung ist der amüsante oder der erbauliche Abglanz der Handelsnachricht. Matteo Bandello, der italienische Erzähler des 16. Jahrhunderts, rechtfertigte bereits die Lust am Fabulieren mit dem Nutzen der Buchführung: »jeden Tag geschehen hübsche Dinge, die man registrieren möchte, so wie die Kaufleute ihren Schriftverkehr festhalten « - ogni giorno avvengono di belle cose, de le quali si vorrebbe tener registro come fanno i mercadanti de le lor scritture. [1] Die Zeit konnte schon dem Florentiner Großhändler des Trecento zu Geld werden, wenn er nur rechtzeitig erfuhr, ob seine eigene Schiffsladung oder die des Konkurrenten den Stürmen, den Piraten zum Opfer gefallen war. Die Kreditgeschäfte mußten, unter dem Druck des kanonischen Zinsverbots, als Währungstausch getarnt werden; so durfte der Bankier die richtige Information über den Wechselkurs des florentinischen Dukaten in London als Gewinn verbuchen. [2] Die Jagd nach den neuesten Nachrichten befriedigte Boccaccio, indem er die Unternehmer über die habgierigen Kurtisanen Palermos aufklärte (Dec. VIII 10). Eine Schicht wohlhabender Großbürger, die die Stadtgesellschaft von Florenz ökonomisch und politisch dirigierte, ermöglichte diese Verkleidung der Handelsnachricht als vergnügliche Novelle. Die Großbürger waren daran interessiert, ihre realen Geschäfte als Schwank zu verharmlosen und sich ihr gesellschaftliches Avancement in die Position des Adels zu bestätigen. Das Ergötzen an derben Handelsinteressen, das Ergötzen an ihrer vielbewährten Tüchtigkeit möchten sie mit aristokratischem Prestige verquicken. Diese Verbindung idealer Normen, die auf die höfische Dichtung zurückweisen, mit der Erfahrung der Wirklichkeit einer bürgerlich städtischen Ordnung versucht Boccaccio im Decameron. Er vermeidet die einfältige Verherrlichung der üblen Gegenwart, indem er die Idee einer harmonischen Stadtgesellschaft, in der kluge Einsicht und Großmut die konkurrierenden Interessen bändigen, in die Vergangenheit entrückt und nur als Erinnerung beschwört. Das ästhetische Ver- 
gnügen über die gegenseitige Betrügerei erinnert jedoch an die Harmonie, die den herrschenden Großbürgern von Florenz in Wirklichkeit nicht gelungen ist. Das edle Kunstschöne, das schon im Decameron einer Oberschicht als Selbstrechtfertigung dient, wird zum Trost für eine vulgäre, glücklose Wirklichkeit. Sie bringt sich aufdringlich in Erinnerung mit der Novellensammlung Franco Sacchettis. Angesichts der glanzvollen Perioden des Decameron könnte man den Terror vergessen, der den Stadtfrieden sicherte. Das vulgäre Chaos des Trecentonovelle soll, als Negativ der boccazesken Harmonie, die fragwürdige Einzigartigkeit des Decameron noch deutlicher hervortreten lassen.

Kunstgenuß als Privileg der Herrschenden wird im Decameron ausdrücklich festgehalten und bleibt unvergessen in der Geschichte der Kunst und Literatur. Boccaccio hat seine Novellen als vergnüglichen Zeitvertreib einer »heiteren Schar" vornehmer Herrschaften gestaltet. Marguerite de Navarre greift auf sein Vorbild zurück, indem sie ihre Erzählungen einer Schar privilegierter Damen und Herren in den Mund legt. Auf Grund einer spezifischen historischen Konstellation nimmt das ästhetische Vergnügen der französischen Höflinge die bitteren Ingredienzien der Gesellschaftskritik, der Moralistik mit auf. Die Poesie als Inbegriff der Gelehrsamkeit und des edlen Vergnügens, wie Cervantes sie darstellen wird, ist aufs innigste an die Herrschaft der Granden gebunden.

"Als das Geld geschaffen wurde, entstand der Betrug" - Come il denaio $f u$ creato, cosi nacque l'inganno, bemerkt gegen Ende des 14. Jahrhunderts einer der desillusioniertesten Erzähler, Franco Sacchetti. [3] Der allgemeinste, von den Fabliaux bis zur italienischen und französischen Erzählung des 16 . Jahrhunderts typische Gehalt der novellistischen Information ist die Verherrlichung des Betrugs, des inganno, und die kluge Nutzung der Profitchancen dank dem ingegno, dem quicken Verstand. Der Geld- und Handelsverkehr der vormanufakturellen Epoche setzte die Auflösung der feudalen Gesellschaftsordnung in Gang, ohne auf ihr äußeres, normatives Gefüge zu verzichten, ohne eigene Lebensformen als Normen der sozialen Ordnung zu proklamieren. [4] Wucher und der freie Wettbewerb des Großhandels entfalteten ihre Energien außerhalb der Zunftregeln und außerhalb des kanonischen Rechts. Mit unrechtmäßigen, aber durchaus tolerierten Mitteln ergaunert sich das Individuum in den Novellen sein Recht. Als Information und als List könnte man Form und Inhalt der Novellistik vom 14. bis zum 16. Jahrhundert verstehen.

Erst Cervantes geht mit seinen Exemplarischen Novellen über die stereotype Darstellung des listigen Betrugs hinaus. Er verzichtet auf neue Variationen der Betrügereien, mit denen das Individuum gegen Sitte und Recht hochzukommen versucht. Die Welt der Gaunerei und die Sphäre edler Rechtschaffenheit treten einander unversöhnlich gegenüber. Ein 
Uberwechseln aus dem einen Bereich in den anderen ist nur im bewußt inszenierten Spiel, auf der Bühne möglich. Wohl war es auch in Spanien zum Konflikt zwischen frühkapitalistischen Tendenzen und der traditionellen Struktur der Gesellschaft gekommen. Aber Cervantes verzichtet auf die Verherrlichung des betrügerischen Triumphs des Einzelnen, der letztlich doch akzeptiert, was er listig hintergeht, weil der unaufhebbare Widerspruch zwischen reich und arm innerhalb der spanischen Gesellschaft des »Goldenen Zeitalters « List und Betrug als Mittel des sozialen Aufstiegs lächerlich erscheinen läßt. Der Betrug garantiert nur innerhalb der Gaunerzunft das Existenzminimum; im bürgerlichen Leben gewährt die List keine Aufstiegschancen. Der Betrug wird im Werk des Cervantes zum Selbstbetrug. Der subjektive Wahn vermag allein noch zu konzipieren, was die Gesellschaft dem Einzelnen versagt. Die unvoreingenommene Information über die spanische Gesellschaft - das scheint Cervantes' Meinung - müßte unweigerlich in Kritik und bittere Satire umschlagen. Ein Erzählen, das auf die Geißelung zeitgenössischer Zustände verzichten und trotzdem die Wahrheit seiner Inhalte bewahren möchte, muß die Einsicht in die Wirklichkeit gleichberechtigt neben die Ansprüche der traditionellen Normen stellen. Nur das ästhetische Spiel zwischen Prosa und Poesie vermag dem Sein und dem Schein gleichermaßen gerecht zu werden. Boccaccio berichtete in schadenfroher Objektivität von glücklichen und unglücklichen Zufällen, ohne das gesellschaftliche System dabei in Frage zu stellen. Das Geschick, das dem Einzelnen widerfährt, wird in Cervantes' besten Erzählungen nicht länger aufgehoben im allgemeinen Sinn einer harmonischen Gesellschaft, der aus der wirklichen abgelesen werden könnte. Die Information über individuelle Schicksale wird in den Exemplarischen Novellen zum Ausdruck des wahnwitzigen, individuellen Widerstands gegen die bestehende Ordnung, aus der ein Entrinnen nicht möglich scheint, oder zum Ausdruck des ergebenen, ohnmächtigen Leidens. Information verwandelt sich in ironisch distanzierte Entlarvung. Betrug schlägt in Selbstbetrug um, in dem sich das Individuum über die Allmacht herrschender Normen wahnhaft hinwegsetzt. Aber die erzählerische Gestaltung dieses Selbstbetrugs soll nicht über die reuige Selbsterkenntnis hinausführen; die gesellschaftlichen Normen sollen nicht angetastet werden. Die fromme Ergebenheit ins Schicksal und das ironische, ästhetische Spiel mit Sein und Schein sind zwei einander bedingende Komponenten in Cervantes' Erzählen.

In der Literaturwissenschaft versuchte man der historischen Parallelität zwischen dem Aufstieg bürgerlicher Schichten und der Entfaltung der Novellistik gerecht zu werden, indem man besonders im Decameron, dem großen Vorbild der europäischen Erzählung, einen "gewissen bürgerlichen Stolz«, kaufmännischen Unternehmungsgeist, die Selbstdarstellung der »bürgerlichen Kultur» wiedererkannte. [5] Die Literaturwissenschaft 
hat es jedoch noch nicht unternommen, die Selbstdarstellung der Kultur in den Erzählungen auf die gesellschaftlichen Interessen hin zu befragen, die sie hervorgebracht haben. Die Kommentare und Interpretationen zu den Novellen haben bisher danach gefragt, wie sie gestaltet seien, ob sie schön oder häßlich, tragisch oder komisch, aristokratisch oder plebejisch auf den Leser wirken. Die Frage nach dem Warum, nach den historischen Gründen der ästhetischen Aussage ging verloren. Jener Begriff der »bürgerlichen Kultur", den man aus dem Decameron so gut wie aus den Erzählungen Maupassants beschwören kann, berücksichtigt weder die besonderen Faktoren der städtischen Ordnung des 14. Jahrhunderts noch ihre ästhetische Vermittlung in den Novellen. Erst der Blick auf die besondere wirtschaftliche und politische Form der Herrschaft kann die historisch relevanten Innervationen der Novellen aufdecken.

Giorgio Padoan hat jüngst den Versuch unternommen, eine Verbindung zwischen dem städtischen Bürgertum und den Erzählungen Boccaccios darzulegen. Er bemerkt, daß eine »bürgerlich-volkstümliche Welt« des Betrugs und der Betrogenen dargestellt wird, daß kluger Utilitarismus und gesunder Bon Sens verherrlicht werden. In diesen »bürgerlichen « Novellen erscheine eine "ganz und gar bürgerliche Vorurteilslosigkeit火. Die »aristokratische Welt« einer anderen Gruppe von Novellen setzt er dagegen $a b$, ohne die bürgerliche Konstruktion dieses aristokratischen Scheins festzuhalten. Vorurteilslos muteten zu ihrer Zeit aber auch die Liebesgedichte Wilhelm IX. von Aquitanien an. [6] Mit dieser literatursoziologischen Kennzeichnung der Novellen, die sich ausschließlich auf das Zeugnis der Kunstprodukte selbst stützt, läßt man gerade die Vorurteile außer acht, die eine Stadtgesellschaft des Trecento mit ihren literarischen Werken zum Ausdruck gebracht hat.

Anregungen für eine soziologische Betrachtung des Heptaméron, der bedeutendsten französischen Novellensammlung des 16. Jahrhunderts, kann man in der Untersuchung von Emile V. Telle, vielleicht auch in dem Hinweis Lucien Febvres finden, daß das Heptaméron ein Erziehungsbuch, ein guide d'honnêteté, der französischen Elite gewesen sei. Ansätze zu einer soziologischen Untersuchung der Exemplarischen Novellen von Cervantes sind gelegentlich vorgelegt worden. Aber die Autoren vergessen entweder die Analyse der Gesellschaft - wie Franz Rauhut - oder die künstlerische Problematisierung dieser Gesellschaft wie Ernesto Francisco Jareño und Ricardo del Arco, die sich beide darauf beschränken, die Milieuschilderungen von Cervantes zu paraphrasieren. Anregungen, Stichworte für eine soziologische Interpretation entdeckt man im Cervantes-Buch von Werner Krauss. Leo Löwenthal liest aus Cervantes' Werk vor allem die Bildung des modernen Bewußtseins, des neuzeitlichen Menschenbildes ab. Die Grundthematik seines Werkes sei »die Autonomie individuellen Denkens und Fühlens«; mit Don Qui- 
jote werde der Mensch, nach dem Zusammenbruch der feudalen Ordnung, als autonomes, moralisch verantwortliches Individuum in das Zentrum der Welt gerückt. Cervantes versuche dabei, die realen Möglichkeiten der Autonomie und ein unerfüllbares, ideales Menschenbild in einem »kritischen Idealismus « zu verbinden. Löwenthal hebt zu Recht einzelne, für den gesellschafts- und bewußtseinsgeschichtlichen Fortschritt bedeutsame Passagen hervor; er geht in seiner Studie nicht auf die ästhetische Struktur einzelner Werke von Cervantes ein. Daher werden auch nicht die historisch bedingten Dissonanzen dieser Werke deutlich, die den »kritischen Idealismus « als fragwürdigen Versuch der Versöhnung verurteilen.

In den vorliegenden Studien über die Erzählungen von Boccaccio, Sacchetti, Marguerite de Navarre und Cervantes strebt der Verfasser nicht der literaturhistorischen Vollständigkeit nach. Die Untersuchung sämtlicher Novellensammlungen der romanischen Literaturen zwischen 1350 und 1600 hätte interessante Variationen des inganno, sogar Darstellungen pathetischen Leidens unmittelbar vor Cervantes - in den Novellen Bandellos - bringen können. Aber die Interpretationen wären mit literarhistorischen Abschweifungen und Querverweisen belastet worden, ohne daß die Fülle des Materials die literatursoziologische Fragestellung gefördert hätte. Der ästhetische Gehalt der Novellistik soll an drei bedeutenden Beispielen, dem Decameron, dem Heptaméron und den Novelas ejemplares, auf seine geschichtliche Bedeutung befragt, der $\mathrm{Zu}-$ sammenhang zwischen Literatur und Gesellschaft soll vor allem an diesen drei Modellen dargelegt werden, weil die Autoren die fragwürdige Ordnung, in der sie lebten, als literarästhetisches Problem begriffen*.

* Die früheren Studien des Verfassers über Boccaccio und Margarete von Navarra (Das Privileg der Lust. Bemerkungen zur Darstellung der irdischen Liebe im Heptaméron; in: Germanisch-Romanische Monatsschrift, NF XVII, 1967, S. 337-353; Aristokratische Händler und käufliche Adlige. Gesellschaftliche Spiegelungen in den Novellen G. Boccaccios und Margaretes von Navarra; in: Gestaltungsgeschichte und Gesellschaftsgeschichte, hg. v. H. Kreuzer u. K. Hamburger, Stuttgart 1969, S. 51-78) sind in überarbeiteter Form in die vorliegende Untersuchung eingefügt worden. Für die kritische Lektüre des Manuskriptes, das der Fakultät für Kultur- und Staatswissenschaften der TH Darmstadt als Habilitationsschrift vorgelegen hat, ist der Verfasser den Herren Prof. Dr. Walter Naumann, Prof. Dr. Helmut Böhme, Prof. Dr. Arcadius R. L. Gurland (Darmstadt), Prof. Dr. Hans-Jörg Neuschäfer (Saarbrücken), Prof. Dr. Erich Köhler und Prof. Dr. Hans-Martin Gauger (Freiburg) zu besonderem Dank verpflichtet; bei der inhaltlichen und stilistischen Uberarbeitung des Manuskriptes konnte er nicht selten von ihren Fragen, Einwänden und Anregungen ausgehen. 